\title{
The Second Source of the Force
}

\author{
Dongkai Li \\ Independent Scholar
}

\begin{abstract}
From the physical science, we know the force comes from the reaction between at least two objects. But, in the reality world, we can see some kind force of an existence does not come from outside. For instance, when a person takes the action such as speaking, movement of arms, we cannot see any force loaded from outside of this person. Then, here is the question: Where does this kind of force come from? This paper is to demonstrate to prove that there is the second source of force, which comes from internal of a kind of object.
\end{abstract}

Keywords: force, existence, contradiction, action, noumenon, person

\section{How Does the Force Come from Internal of an Independent Existence?}

The noumenon refers to the object which is the basis and initial for everything. The basis for everything means that the existence of the noumenon relies on nothing. The initial for everything means that the noumenon is the first in any way, relying on nothing. In this way, we could say that the noumenon is an existence relying on nothing.

"Rely on" means that there should be a kind of force affection performing the function between two things at least. "Rely on nothing" means the force needed by an existence comes from this existence only, not from others. Here, we could say that the noumenon should produce force by itself.

For an absolute static status, there should be no force. Since the noumenon could produce the force by itself, we should say the static should not be a nature of the noumenon, and the noumenon contains more than one unit within itself.

The force from others means there is a unit receiving the force and other unit delivering the force. For the receiver, the force should come from one unit at least.Then we should say the noumenon should have two aspects within itself, one delivering the force, the other one receiving the force.

These two aspects shall never have the possibility to be changed into one single aspect. For this purpose, these two aspects should have a nature of contradiction. Here, we finally come to a conclusion that the noumenon is an existence with a pair of contradiction within its body.

There are many kinds of contradiction. Since the noumenon relies on nothing, the contradiction within the noumenon is not a kind of specific contradiction. This means the contradiction within the noumenon is just a pair of contradiction.

The noumenon relies on nothing, so, there is no material in the noumenon, which means the noumenon is a pure modality of a pair of contradiction.

Dongkai Li, independent scholar, China; main research field: Ontology, Cognition, and People.

The author ever attended the 22nd and 23rd World Congress of Philosophy with his 6 papers approved for discussion by the Congress. His published philosophy book is What Is the Existence by XUELIN ISBH. 
An existence containing a pair of contradiction within its body could produce the force for sustaining the existence. This kind force comes from internal of an independent existence. This is the second source of the force.

In this way, such an existence could be independent. So, any object could be distinguished as an independent existence or not. If one object has a pair of contradiction producing the force for itself, its existence could be independent; if not, it is not an independent object, but a kind of accessory belonging to other independent existence.

\section{How Does a Person Produce the Force within the Body?}

The first condition for a person's living is the satisfaction of his or her natural body demand. There are two aspects involved for the satisfaction of the natural demand: One is the healthy body with its natural demand function system; the other is the object in the world to serve the demand.

The healthy natural demand function system is in the person's body, and the objective thing to serve the demand is outside of the body. Here is a question, how does the person take the outside object to serve his body's demand? Let us check the process of person's action of looking for the object for serving his body demand.

When "I" had the demand for water, my body function would make me feel thirsty. But, the water is not in my body. In my body, I have no water to serve this demand. I must take action looking for the water in the nature.

What I need is the water, not stone, not the bread. So, I must confirm the objective water is the water wanted.

This judgment was made by comparing the objective object with the knowledge or idea in person's consciousness.

If there is the idea or image about the water in my consciousness, when I see the water, I would check this objective water with this idea. If the object complying to this idea, then, I would confirm that object is the one I want.

If I do not have the idea about the water, then, I cannot confirm an object as water or not. For example, if you do not know a person, then, you would not find him even if he is just standing at your side.

So, we would say without the knowledge or ideas in a person's consciousness, the person would not find the object to serve his body demand. The consciousness is in a person's body, but, these idea or knowledge in the consciousness cannot serve the demand of the body. Here is a contradiction in a person, the demand of the body and the idea in the consciousness. Here, I call the natural body with its demand function system as "ego," and the idea refers to the outside objective thing in the consciousness as "Nonego." The "Ego" and the "Nonego" is a pair of contradiction within a body, producing the force for movement of a person.

Any living thing such as animal and the plant has this kind of contradiction producing the force within the body.

\section{Works Cited}

Dongkai, Li. What Is the Existence.

Dongkai, Li. A Breakthrough in the Logical Deduction about the Cognition Theory. 